\title{
L-Arginine supplementation in severe asthma
}

\author{
Shu-Yi Liao, ${ }^{1,2}$ Megan R. Showalter, ${ }^{3}$ Angela L. Linderholm, ${ }^{1}$ Lisa Franzi, ${ }^{1}$ Celeste Kivler, ${ }^{4}$ Yao Li, ${ }^{5}$ \\ Michael R. Sa, ${ }^{3}$ Zachary A. Kons, ${ }^{3}$ Oliver Fiehn, ${ }^{3}$ Lihong Qi, ${ }^{5}$ Amir A. Zeki, ${ }^{1,2}$ and Nicholas J. Kenyon ${ }^{1,2}$ \\ 'Division of Pulmonary, Critical Care, and Sleep Medicine, Department of Internal Medicine, UCD, Sacramento, California, \\ USA. ${ }^{2}$ VA Northern California Health Care System (VANCHCS), Mather, California, USA. ${ }^{3} \mathrm{NIH}$ West Coast Metabolomics \\ Center, ${ }^{4}$ Department of Respiratory Therapy, and ${ }^{5}$ Department of Public Health Sciences, UCD, Davis, California, USA.
}

BACKGROUND. Dysregulation of L-arginine metabolism has been proposed to occur in patients with severe asthma. The effects of L-arginine supplementation on L-arginine metabolite profiles in these patients are unknown. We hypothesized that individuals with severe asthma with low fractional exhaled nitric oxide (FeNO) would have fewer exacerbations with the addition of L-arginine to their standard asthma medications compared with placebo and would demonstrate the greatest changes in metabolite profiles.

METHODS. Participants were enrolled in a single-center, crossover, double-blind L-arginine intervention trial at UCD. Subjects received placebo or L-arginine, dosed orally at $0.05 \mathrm{mg} / \mathrm{kg}$ (ideal body weight) twice daily. The primary end point was moderate asthma exacerbations. Longitudinal plasma metabolite levels were measured using mass spectrometry. A linear mixed-effect model with subject-specific intercepts was used for testing treatment effects.

RESULTS. A cohort of $\mathbf{5 0}$ subjects was included in the final analysis. L-Arginine did not significantly decrease asthma exacerbations in the overall cohort. Higher citrulline levels and a lower arginine availability index (AAI) were associated with higher FeNO $(P=0.005$ and $P$ $=2.51 \times 10^{-9}$, respectively). Higher AAI was associated with lower exacerbation events. The eicosanoid prostaglandin $\mathrm{H}_{2}\left(\mathrm{PCH}_{2}\right)$ and $\mathrm{N}_{\alpha}$-acetyl-L-arginine were found to be good predictors for differentiating clinical responders and nonresponders.

CONCLUSIONS. There was no statistically significant decrease in asthma exacerbations in the overall cohort with L-arginine intervention. $\mathrm{PCH}_{2}, \mathrm{~N}_{\alpha}$-acetyl-L-arginine, and the AAI could serve as predictive biomarkers in future clinical trials that intervene in the arginine metabolome.

TRIAL REGISTRATION. ClinicalTrials.gov NCT01841281.

FUNDING. This study was supported by NIH grants R01HL105573, DK097154, UL1 TR001861, and K08HL114882. Metabolomics analysis was supported in part by a grant from the University of California Tobacco-Related Disease Research Program program (TRDRP).

Authorship note: SYL and MRS are co-first authors.

Conflict of interest: The authors have declared that no conflict of interest exists.

Copyright: () 2020, American Society for Clinical Investigation.

Submitted: March 3, 2020

Accepted: May 27, 2020

Published: July 9, 2020.

Reference information: JCI Insight. 2020;5(13):e137777.

https://doi.org/10.1172/jici.

insight.137777.

\section{Introduction}

The measurement of exhaled breath NO is recommended to assist in phenotyping, and perhaps diagnosing, patients with asthma (1-3). NO is a product of L-arginine conversion to L-citrulline via the NOS enzyme isoforms. We and others have found that mice exposed to aeroallergens have limited bioavailability of the substrate for NOS, L-arginine, in the airway compartment (4-7). Similarly, arginine metabolic endotypes have been identified in severe asthma, with a subset of patients with high fractional exhaled NO (FeNO) demonstrating increased levels of arginine turnover and worse clinical outcomes. The potential to identify patients who may benefit from supplementation with L-arginine or perhaps L-citrulline, the primary endogenous pool of L-arginine, based on a predictive FeNO value is intriguing (8).

Dysregulation of arginine metabolism and depletion of key arginine metabolites constitute a paradigm linked to multiple diseases, including obesity, metabolic syndrome, and asthma $(9,10)$. This phenomenon is also the driving hypothesis in numerous clinical trials in cardiovascular disease $(11,12)$ and sickle cell disease (13). From these studies, we have learned that there are other factors to consider, such 


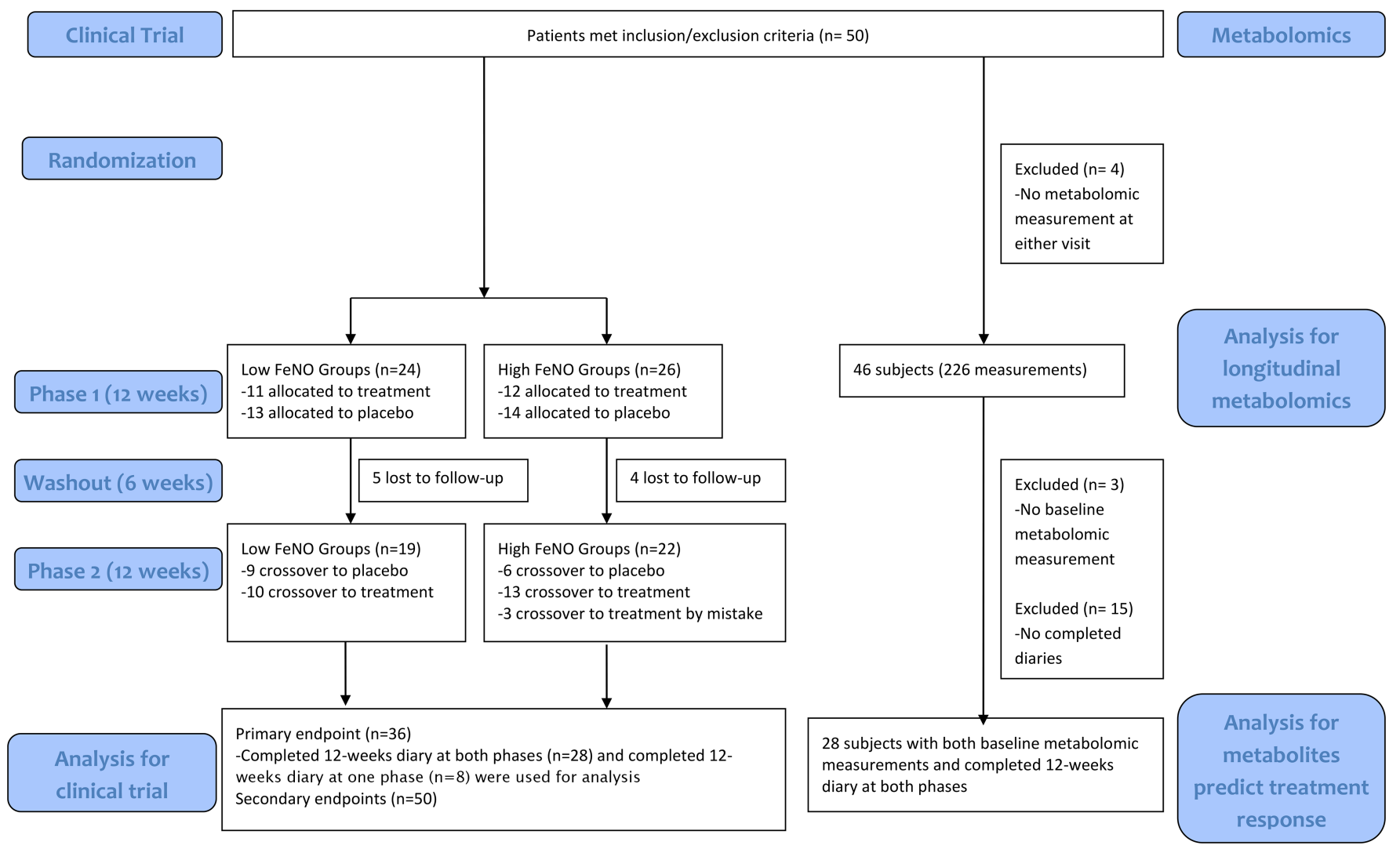

Figure 1. Consort diagram of L-arginine study. The diagram depicts patient flow through clinical trial and metabolomics study.

as asymmetric dimethylarginine (ADMA), which can accumulate and lead to impaired NO production (14-16). ADMA is a potent NOS inhibitor that is formed through a posttranslational modification of $\mathrm{L}$-arginine and is metabolized to L-citrulline and dimethylamine.

A recent clinical trial of L-citrulline supplementation in individuals with asthma found that L-citrulline increased FeNO and modestly improved forced expiratory volume in 1 second ( $\left.\mathrm{FEV}_{1}\right)$, especially in females with late-onset asthma (17). We and others have found in murine models that manipulation of L-arginine content in the airway compartment via L-arginine treatment or inhibition of the arginase enzyme decreases airway inflammatory cell counts, lung lavage cytokine levels, airways hyperresponsiveness, and arginase activity (18-20). We hypothesized that patients with severe asthma on controller medications with a low or normal FeNO would have fewer asthma exacerbations when supplemented with L-arginine over a 3-month period compared with patients with high FeNO. To address this hypothesis, we performed a single-center randomized clinical trial of L-arginine supplementation in patients with severe asthma grouped according to FeNO level. We also integrated a longitudinal metabolomic analysis to further provide insights into metabolic impacts of disease pathogenesis and to potentially identify biomarkers of responders to this therapy.

\section{Results}

Effects of L-arginine supplementation on clinical endpoints. We randomized a total of 50 subjects, with 24 subjects in the low-FeNO group (FeNO $<20$ parts per billion [ppb]) and 26 patients in the-high FeNO group (FeNO $>25$ $\mathrm{ppb}$ ). In phase 1, approximately one-half of the patients were allocated to L-arginine treatment. The withdrawal of subjects during the study was similar regardless of the randomization and FeNO group. Unfortunately, 3 subjects in the high FeNO group were allocated to the initial treatment again during the phase 2, and this was disclosed at the time it was discovered by the UCD Investigational Drug Service (IDS). The consort diagram is shown in Figure 1. Study enrollment began August 2013, with final data collected for the primary end point in August 2018 and all metabolomics data collected by December 2019. 
Table 1. Characteristics of patients in the study

\begin{tabular}{|c|c|c|c|c|}
\hline Variable names & Total $(n=50)$ & Low FeNO $(n=24)$ & High FeNO $(n=26)$ & $\boldsymbol{P}$ \\
\hline $\begin{array}{l}\text { White/African American and } \\
\text { Asian (\%) }\end{array}$ & $78.0 / 22.0$ & $79.2 / 20.8$ & $76.9 / 23.1$ & 1.00 \\
\hline Age (yr) & $54.3 \pm 13.2$ & $56.5 \pm 11.9$ & $52.4 \pm 14.3$ & 0.27 \\
\hline BMI $\left(\mathrm{kg} / \mathrm{m}^{2}\right)$ & $33.6 \pm 8.4$ & $35.3 \pm 7.0$ & $32.2 \pm 9.4$ & 0.19 \\
\hline Weight (kg) & $90.0 \pm 24.5$ & $94.8 \pm 20.5$ & $85.6 \pm 27.3$ & 0.19 \\
\hline $\mathrm{FVC}(\mathrm{L})$ & $2.7 \pm 1.0$ & $2.6 \pm 0.9$ & $2.8 \pm 1.1$ & 0.48 \\
\hline FVC (\% predicted) & $77.7 \pm 21.1$ & $74.2 \pm 18.4$ & $80.8 \pm 23.2$ & 0.28 \\
\hline $\mathrm{FEV}_{1} / \mathrm{FVC}(\%)$ & $73.5 \pm 12.0$ & $70.1 \pm 13.7$ & $76.4 \pm 0.10$ & 0.07 \\
\hline ACT score & $16.1 \pm 5.0$ & $16.0 \pm 4.6$ & $16.1 \pm 5.5$ & 0.96 \\
\hline
\end{tabular}

All continuous variables are presented as mean \pm SD.

Subjects in the low-FeNO group at baseline had demographic characteristics compared similar to those in the high FeNO group (Table 1). The average age of all subjects was $54.3 \pm 13.2$ years, they were predominantly female $(76 \%)$, obese (BMI $33.6 \pm 8.4$ ), and had asthma control test (ACT) score averages of 16.1 \pm 5.0 , suggesting poor asthma control. Perhaps most interesting is the apparent difference in mean $\mathrm{FEV}_{1}$ percent predicted between the 2 groups. For all subjects, mean $\mathrm{FEV}_{1}$ was $73.1 \% \pm 23.9 \%$, but it showed a trend to be lower in the low-FeNO group $(66.7 \% \pm 20.7 \%)$ compared with the high-FeNO group $(88.8 \% \pm$ $25.4 \% ; P=0.07)$. This difference was not statistically significant but might be important clinically. It was unexpected and is not explained by other demographic factors.

The $\mathrm{FEV}_{1} / \mathrm{FVC}$ ratio had a similar pattern: the low-FeNO group had a trend toward a lower ratio $(0.70$ $\pm 0.14)$ compared with the high-FeNO group $(0.76 \pm 0.001, P=0.07)$. Importantly, there was no statistically significant carryover or period effect on any of the primary or secondary outcomes. For the primary outcome, there was no significant treatment effect on the reduction in the exacerbation events $(P=0.41)$. The treatment effects were not affected by the FeNO group $(P=0.78)$. Similarly, no treatment effects were observed on the secondary outcomes, including changes in ACT scores, $\mathrm{FEV}_{1}, \mathrm{FVC}, \mathrm{FEV}_{1} / \mathrm{FVC}(\%)$, or weight. However, the interaction term "treatment $\times$ FeNO" showed a statistically significant difference for both absolute FVC and for FVC percent predicted ( $P=0.02$ for both measures). Specifically, this was significant when comparing the low-FeNO with the high-FeNO group $(P<0.001)$. With L-arginine treatment, the low-FeNO group had a significant, 0.45 -L (or $0.12 \%$ predicted) increase in FVC compared with the FVC in the high-FeNO group (Table 2). Also, while L-arginine treatment did not have a direct effect on FeNO for the entire cohort, in the low-FeNO group, FeNO levels increased significantly more compared with those in the placebo group ( $\beta$ estimate $=-7.1, P=0.02$, data not shown), suggesting an expected treatment effect.

Metabolites that differentiate subgroups. Forty-three subjects had metabolites collected and measured at first visit. Several metabolites, such as mannose and cystine, demonstrated good performance in discriminating low- versus high-FeNO status, with the variable importance in projection (VIP) score greater than 2.6 using the partial least squares discriminant analysis (PLS-DA) algorithm (Figure 2A). The same analytic approach was used to understand the effect of FeNO and treatment status at the last visit of each intervention. This analysis showed multiple metabolites with high VIP scores (Figure 2B) that were different between L-arginine treatment and placebo. Finally, dipeptide- and arginine-related metabolites that differentiate FeNO/ treatment status using hierarchical cluster analysis (presented as a heatmap) are described below.

Baseline metabolites that predicted arginine treatment response. To investigate whether any metabolites could predict a clinical response to treatment, we examined the subgroup of 28 subjects who had a complete daily diary, study visit, and metabolic data set. Among this subgroup of subjects, we performed a more focused post hoc analysis to determine whether baseline predictive metabolites could be identified that might suggest a clinical response predictor to L-arginine. We noted that 8 individuals responded to the L-arginine treatment as defined by a clear reduction in exacerbation events of at least $33 \%$. All those 28 subjects were included in the downstream analysis. 
Table 2. Testing for interaction between treatment and FeNO group using mixed-effect model

\begin{tabular}{|c|c|c|c|c|c|c|}
\hline End points & Treatment (estimate) & Treatment $(P)$ & $\begin{array}{l}\text { FeNO (ref: high) } \\
\quad \text { (estimate) }\end{array}$ & FeNO (P) & $\begin{array}{l}\text { Treatment } \times \text { FeNO } \\
\text { (estimate) }\end{array}$ & Treatment $\times$ FeNO $(P)$ \\
\hline Exacerbation events & 0.17 & 0.41 & 0.04 & 0.90 & $N A^{A}$ & 0.78 \\
\hline ACT score & 0.34 & 0.74 & -0.32 & 0.67 & -0.37 & 0.71 \\
\hline ACT change & -0.30 & 0.86 & -1.25 & 0.51 & 0.19 & 0.94 \\
\hline $\mathrm{FEV}_{1}$ change & -0.13 & 0.31 & 0.08 & 0.56 & 0.23 & 0.21 \\
\hline FEV,$\%$ change & -0.04 & 0.35 & 0.03 & 0.53 & 0.08 & 0.21 \\
\hline $\mathrm{FEV}_{1} / \mathrm{FVC}$ change & 0.04 & 0.12 & 0.04 & 0.09 & -0.06 & 0.11 \\
\hline Weight change & 0.54 & 0.61 & -0.44 & 0.70 & -0.25 & 0.87 \\
\hline
\end{tabular}

${ }^{A}$ Not available: The estimate was not provided by the SAS GLIMMIX procedure. Bold type indicates significant $P$ values for treatment $\times$ FeNO status.

In this analysis, several unexpected predictive metabolites were found, including prostaglandin $\mathrm{H}_{2}$ $\left(\mathrm{PGH}_{2}\right)$, which demonstrated good performance in discriminating the treatment responder group (VIP score of 2.61 by PLS-DA algorithm). We should note that we cannot be fully confident that the species we are annotating is solely $\mathrm{PGH}_{2}$, as the chromatography method used was not designed specifically for prostaglandin analysis, and an authentic $\mathrm{PGH}_{2}$ standard was not run. However, closely related prostaglandin standards had similar retention times, and our experimental spectra matched findings from others (21). Further validation with a targeted prostaglandin method is needed to fully identify this compound, whose peak and mass spectral data are outlined in Supplemental Figure 1; supplemental material available online with this article; https://doi.org/10.1172/jci.insight.137777DS1.

Another important metabolite, $N_{\alpha}$-acetyl-L-arginine, also had a high VIP score at 2.21. Other key predictive metabolites are shown in Figure 3A. The baseline intensity of $\mathrm{PGH}_{2}$ was found to be higher, while the intensity of $N_{\alpha}$-acetyl-L-arginine was found to be lower in the treatment response group (Figure 3A). A different approach using hierarchical cluster analysis (presented as a heatmap) discovered several overlapping metabolites that were also identified using the PLS-DA method (Figure 3B). The number of dipeptides identified with this method was surprising. Given the increasing interest in the role peptidases, particularly dipeptidyl peptidase-4 (DPP4) $(22,23)$, might play in asthma, we performed a more complete analysis of these compounds. Dipeptide- and arginine- related metabolites that predict treatment response using hierarchical cluster analysis (presented as a heatmap) are shown in Figure 4B. Treatment responder versus nonresponder groups had similar clinical characteristics overall (data not shown), except that the average weight in the treatment response group was higher $(106.8 \mathrm{~kg}$ versus $86.1 \mathrm{~kg}, P=0.04)$, although there was no significant difference in BMI $(P=0.07)$. No significant differences were observed regarding lung function, FeNO, or ACT scores.

Longitudinal metabolomic profiling during the study showed temporal differences in profiles between treatment groups. Forty-six subjects with 226 metabolomic measurements (average 4.9 measurements per subject) were used for the complete metabolomic profiling (Table 3). Notably, a higher level of citrulline, the primary pool of endogenous L-arginine, and lower arginine availability index (AAI) - defined as arginine/(ornithine + citrulline) - was associated with higher FeNO, with $P=0.005$ and $P=2.51 \times$ $10^{-9}$, respectively. This suggests that L-citrulline rather than L-arginine plasma concentration is a better measure of the substrate pool to produce NO in the airway compartment. In addition, a higher AAI was associated with lower exacerbation events $(P=0.02)$. For the time-series analysis with an untargeted metabolomics approach, the top 10 metabolites with different temporal profiles in treatment versus placebo groups are shown in Table 4 . Arginine metabolites such as L-arginine, $N_{\alpha}$-acetyl-L-arginine, and ornithine were among the top 10 metabolites of a total of 542 metabolites evaluated.

The patterns of those metabolites plotted with visits are shown in Figure 5. They demonstrate an increase in arginine, $N_{\alpha}$-acetyl-L-arginine, and ornithine during the treatment period compared with the placebo period, all of which suggest that the L-arginine dose was measurable and reasonable. Other arginine-related metabolites were ranked as follows: ADMA, 273; SDMA, 513; urea, 112; and citrullin, 219. These metabolites did not change with the treatment, although no $P$ value was reported using the method of multivariate empirical Bayes (MEBA) time-series analysis (24). The top 10 metabolite 
A

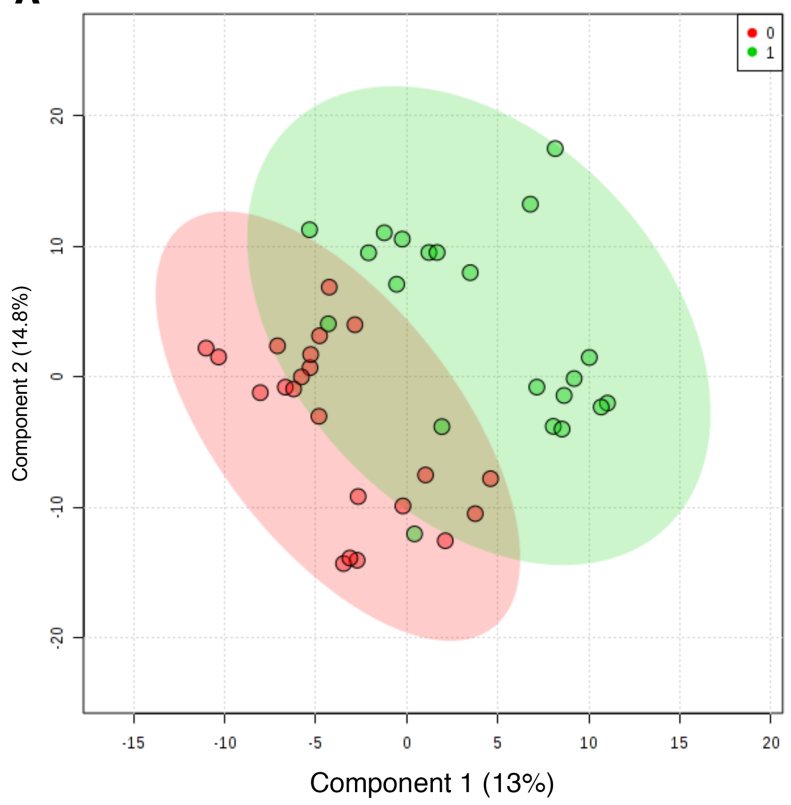

$\mathbf{B}$

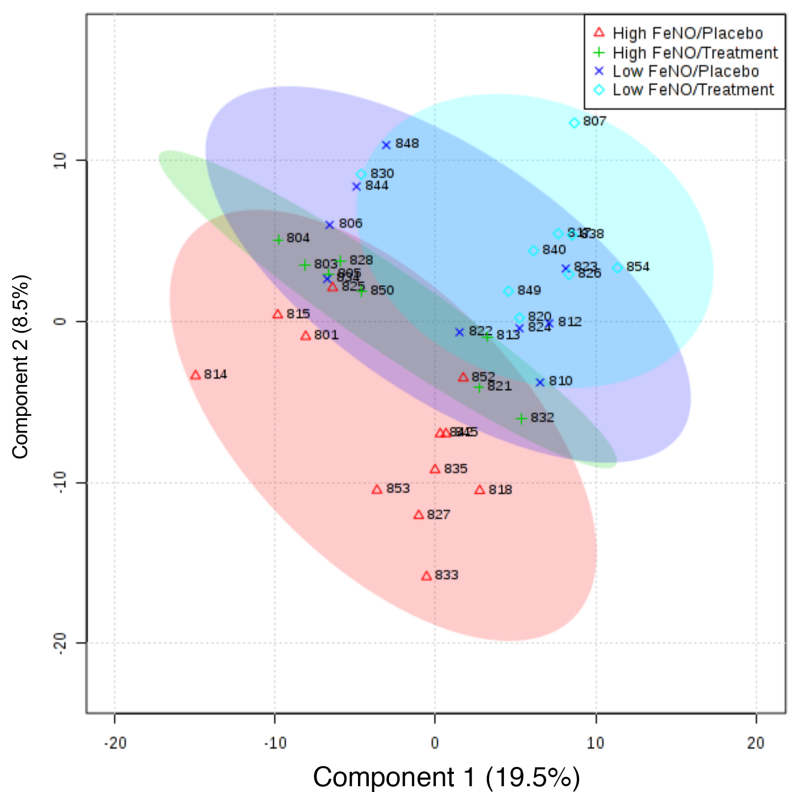

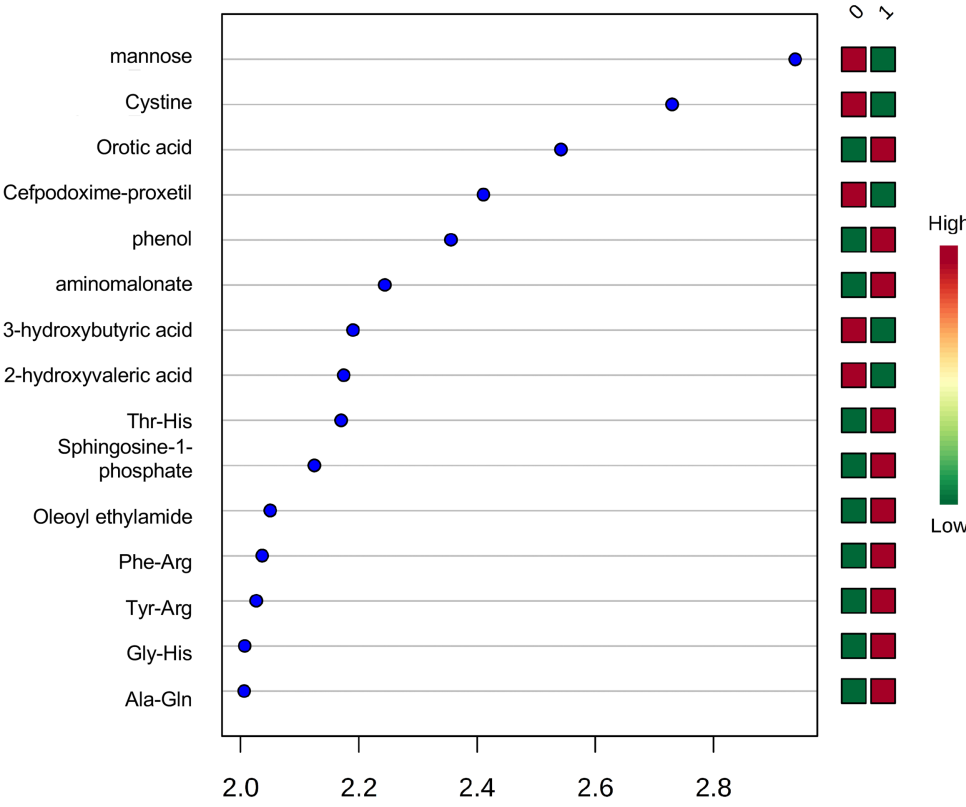

VIP scores

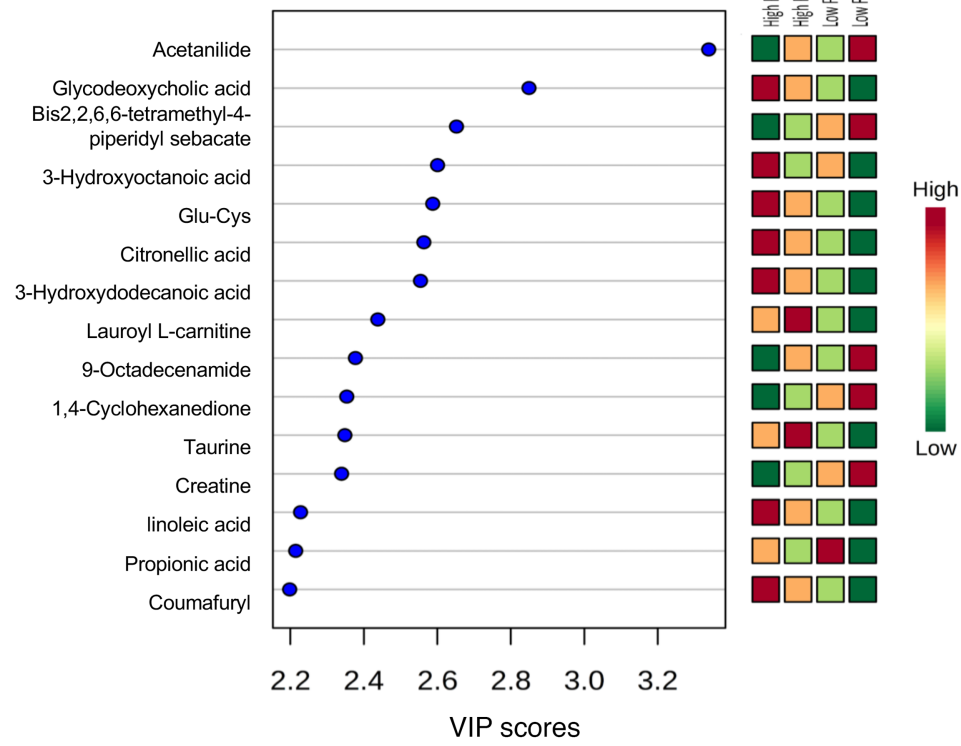

Figure 2. Plasma metabolites by FeNO group and by L-arginine treatment status. (A) Top metabolites (baseline metabolites) that differentiate high-FeNO ( $n=21)$ (1) versus low FeNO $(n=22)$ (0) subjects using PLS-DA. (B) Top metabolites (metabolites at visit 3) that differentiate FeNO/treatment status using PLS-DA. Numbers in the left panel are participant identifiers.

pattern changes in other biological conditions, including (a) high- versus low-FeNO group during the treatment period and (b) treatment response versus nonresponse group, are shown in Tables 5 and 6 .

Adverse events. L-Arginine and the placebo pills were generally well tolerated. Adherence to therapy was confirmed based on measurement of L-arginine and arginine metabolites in blood and by pill counts. There were 4 hospitalizations due to asthma exacerbations in the clinical trial: 2 during the intervention period, 1 during the washout period after the intervention, and 1 during the placebo period. Three subjects withdrew upon mutual agreement for either pill dysphagia or hives. Of the 2 with dysphagia, one was taking L-arginine at the time. The subject with hives was also taking L-arginine. Overall, the side effect profile of L-arginine was considered low by the investigators. 
A

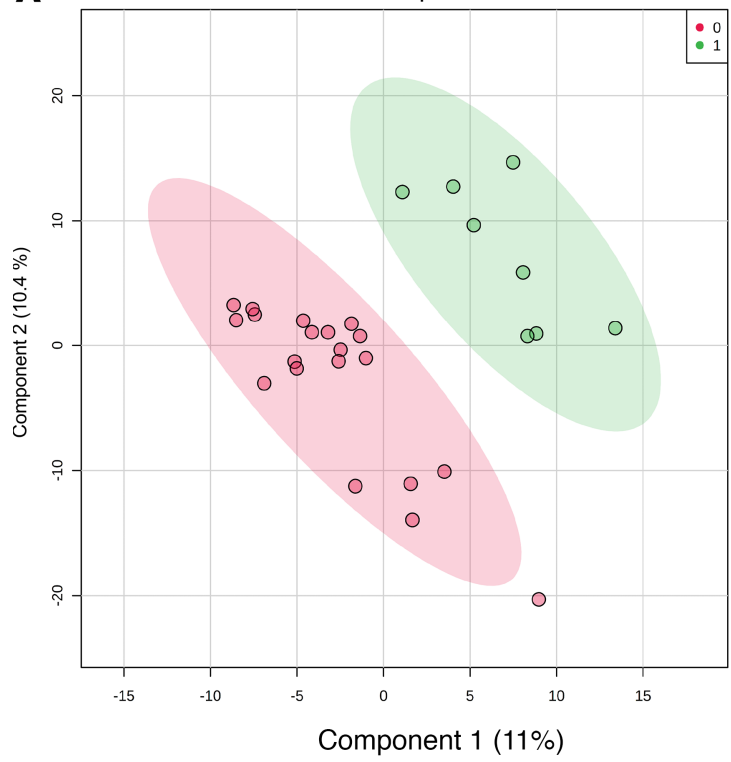

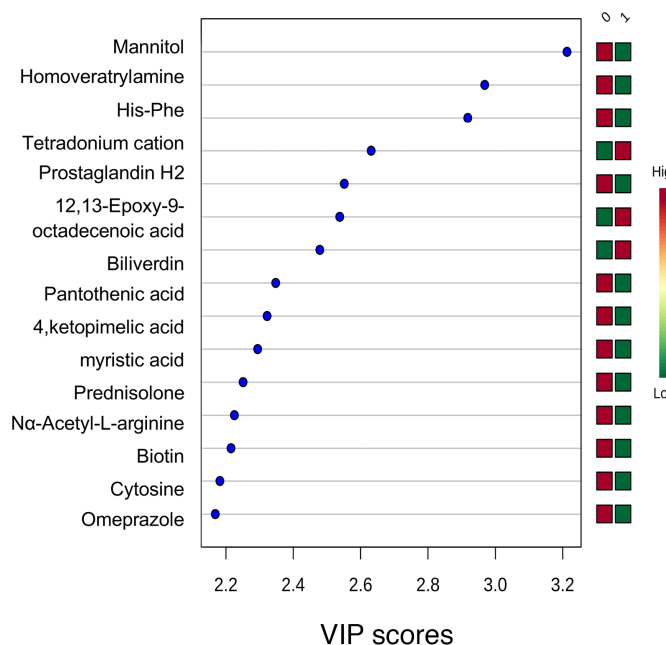

Prostaglandin $\mathrm{H} 2$ intensity (autoscale)

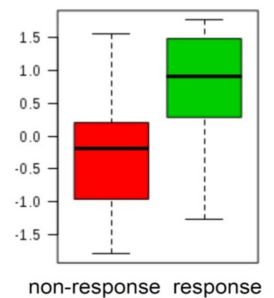

Na-Acetyl-L-Arginine intensity (autoscale)

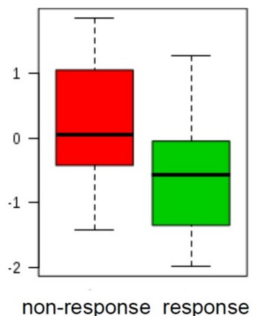

B

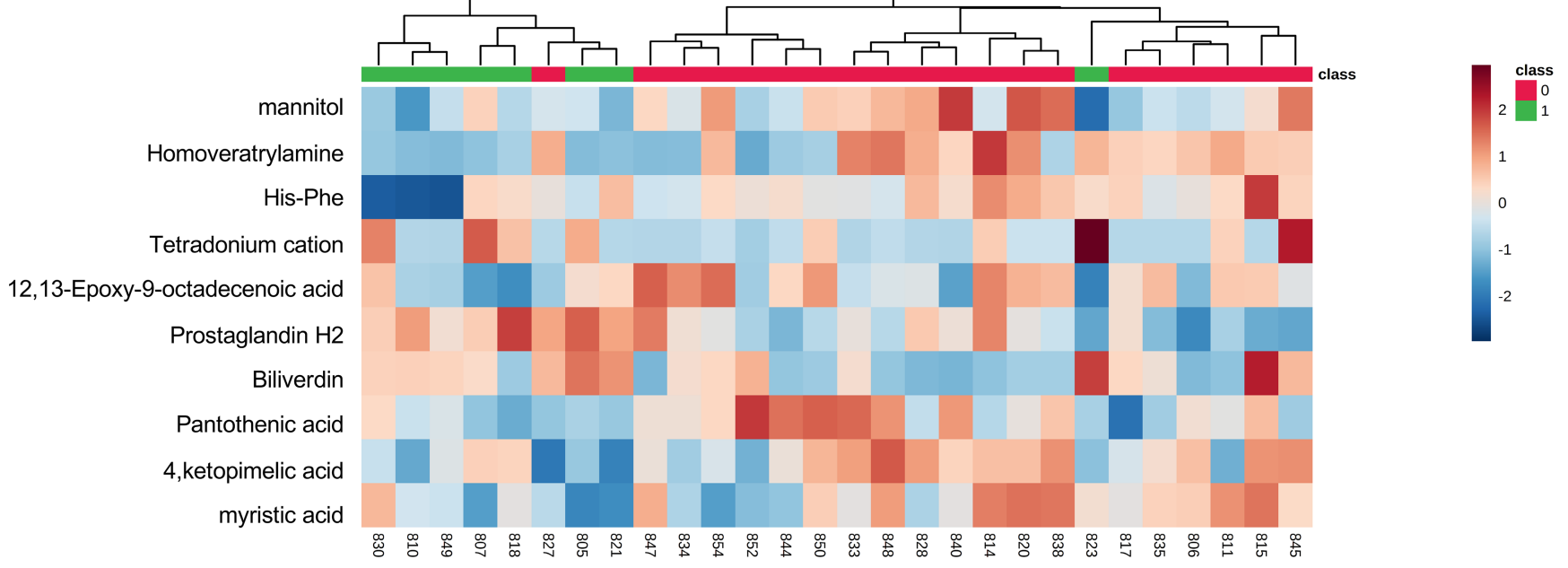

Figure 3. Metabolites predicting response to L-arginine. (A) Score plot; top metabolites that predicted treatment response using PLS-DA ( $n=28$ total); and $\mathrm{PCH}_{2} / \mathrm{N}_{\alpha}$-acetyl-L-arginine levels in the treatment response (green, 1) versus nonresponse group (red, 0). (B) Top metabolites that predict treatment response using hierarchical cluster analysis. For the metabolite intensity, red indicates higher intensity, while blue indicates lower intensity. For example, participants in the treatment response group (green, 1) have a higher prostaglandin level (mostly red).

\section{Discussion}

A paradigm of dysregulated arginine metabolism has recently been proposed in severe asthma, suggesting that subsets of severe asthma patients may respond differently to, and benefit from, supplementation with the amino acid L-arginine. The same could hold true for $\mathrm{L}$-citrulline, which is the primary store of $\mathrm{L}$-arginine in humans. This paradigm derives from years of preclinical and clinical studies in heart, blood, and lung diseases suggesting that $\mathrm{L}$-arginine metabolism is disturbed during periods of metabolic and inflammatory stress.

Our rationale for pursuing $\mathrm{L}$-arginine as a possible therapy in asthma is that L-arginine is an inexpensive, safe, and readily available supplement. L-Arginine could be used, therefore, as a cost-effective add-on therapy to a standard controller regimen with minimal side effects. In this setting, we would argue that a clinical response rate of even $20 \%$ is meaningful when treating these complex patients on multimodal therapy. To address this clinical question in asthma, we designed a unique, single-center clinical trial of L-arginine supplementation that integrates a longitudinal metabolomics analysis with clinical outcomes. Our principal findings can be summarized as follows: (a) we did not identify any significant clinical benefits of L-arginine 

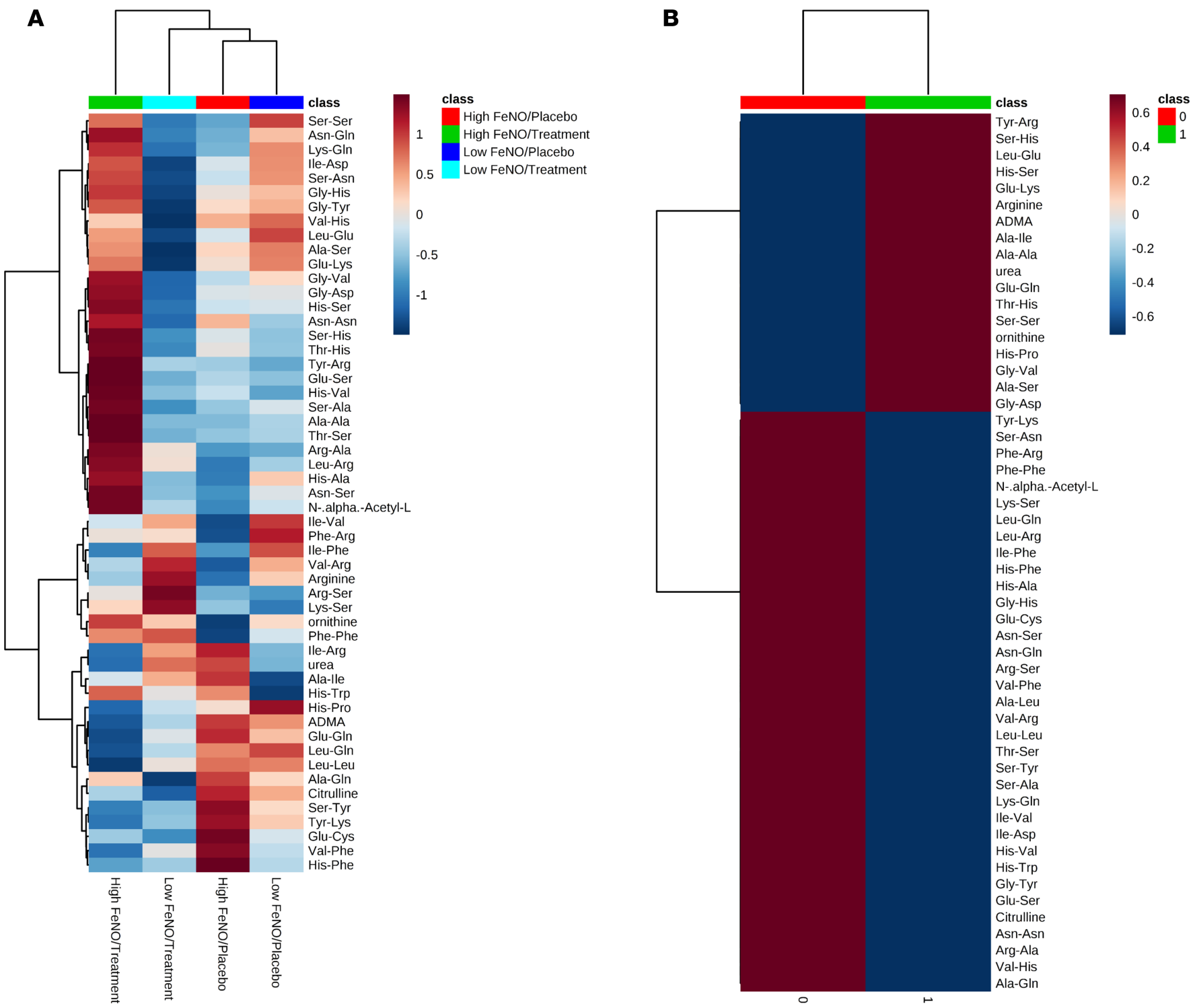

Figure 4. Plasma dipeptide metabolites among study groups. (A) Dipeptide- and arginine-related metabolites that differentiate FeNO/treatment status $(n=43)$ using hierarchical cluster analysis. For metabolite intensity, red indicates higher intensity, while blue indicates lower intensity. (B) Dipeptide- and arginine-related metabolites that predict treatment response (green, 1 ) versus nonresponse group (red, 0$)$ using hierarchical cluster analysis ( $n=28$ ). For the metabolite intensity, red indicates higher intensity, while blue indicates lower intensity.

supplementation in the individuals with severe asthma we randomized other than change in FVC; (b) serum arginine metabolites collected in this longitudinal study support the proposed arginine endotype paradigm; and (c) we identified serum metabolites that could potentially predict treatment response.

Using a rigorous metabolomics approach, we demonstrated that the addition of L-arginine to standard-of-care asthma medications increased L-arginine and $N_{\alpha}$-acetyl-L-arginine content. This result strongly suggests that the intervention of oral L-arginine was appropriate and delivered at a reasonable dose, as the increase in serum arginine-related metabolite levels was sustained during the intervention period. There was no clinical benefit of L-arginine with regard to asthma exacerbations in either the low- or high-FeNO groups. The hypothesis that individuals with asthma having low FeNO at baseline would derive the most benefit from L-arginine supplementation was rejected with respect to the primary clinical outcome. Our main hypothesis was based on a series of preclinical and clinical studies (6, 14, $25,26)$ suggesting that adequate supplementation with L-arginine substrate (for the NO synthase pathway) could ameliorate inflammation and improve airway hyperresponsiveness. However, given the more recent evidence that L-arginine turnover may be more enhanced in high-FeNO groups and in individuals 
Table 3. Associations between the outcomes and intensities of arginine metabolism-related metabolites

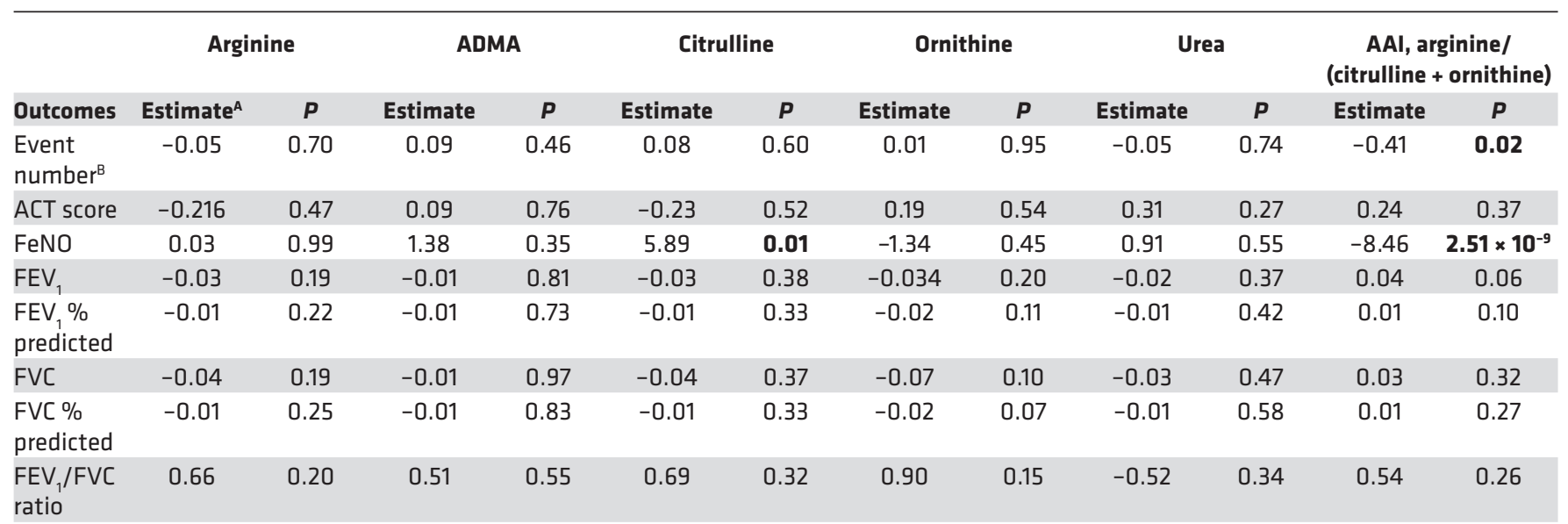

${ }^{A}$ Estimates gave only the direction of the effects of metabolites, since the level is the intensity, not the absolute concentration. All the intensities were autoscaled. ${ }^{B}$ Event number in 12 weeks was calculated at the last visit of the 12-week periods; therefore, only the measurements at visits 3 and 6 were included in the analysis. Bold type indicates significant $P$ values for the association tests

with ARG2 variants and that the arginine metabolome is affected by environmental factors such as traffic pollution, the original hypothesis may have been misguided $(27,28)$.

Despite the negative result overall, we did identify some interesting predictive baseline metabolic biomarkers that have implications for proper patient selection for L-arginine therapy. This is the first study of which we are aware that employed a machine learning algorithm to identify a biomarker panel to predict response to L-arginine. Although the arginine level itself was not a predictive biomarker for treatment response, a low baseline $N_{\alpha}$-acetyl-L-arginine level was. Therefore, subjects with low baseline $N_{\alpha}$-acetyl-L-arginine levels may respond to arginine supplementation.

Another top predictive metabolite, $\mathrm{PGH}_{2}$, is also of interest. The intensity of $\mathrm{PGH}_{2}$ was found to be higher in the L-arginine treatment response group. $\mathrm{PGH}_{2}$ is the upstream product of $\mathrm{PGE}_{2}$ and $\mathrm{PGF}_{2 \alpha}$. In a previous study of individuals with asthma, both inhaled $\mathrm{PGE}_{2}$ and $\mathrm{PGF}_{2 \alpha}$ were shown to reduce FeNO (26). One possible explanation for why patients with high baseline prostaglandins benefited from the L-arginine supplement is that L-arginine can restore the effect of prostaglandins on NOS in those populations, but it may not add any additional beneficial effect for those with normal NOS function (low-prostaglandins population).

In addition, a remarkable number of dipeptides differed between responder groups in a secondary analysis. A key dipeptide peptidase, DPP4 (CD26), is of interest in the pathophysiology of several lung diseases, including pulmonary hypertension and asthma $(29,30)$. DPP4 is a transmembrane exopeptidase that cleaves dipeptides from the N-terminus of cytokines, chemokines, and incretins such as glucagon-like peptide 1 (GLP1). It is expressed in immune and airway epithelial cells, and circulating levels

Table 4. Top natural metabolites with different temporal profiles in treatment versus placebo groups

\begin{tabular}{cc}
\hline Metabolites & ${\text { Hotelling's } \mathbf{T}^{\mathbf{2}}}$ \\
\hline$N_{\alpha}$-acetyl-L-arginine & 11.20 \\
$N$-methyllysine & 9.54 \\
Kynurenic acid & 8.56 \\
Arginine & 8.28 \\
\hline Hydroxycarbamate & 7.93 \\
\hline Ornithine & 7.73 \\
\hline 2-Phenylacetamide & 7.69 \\
\hline
\end{tabular}



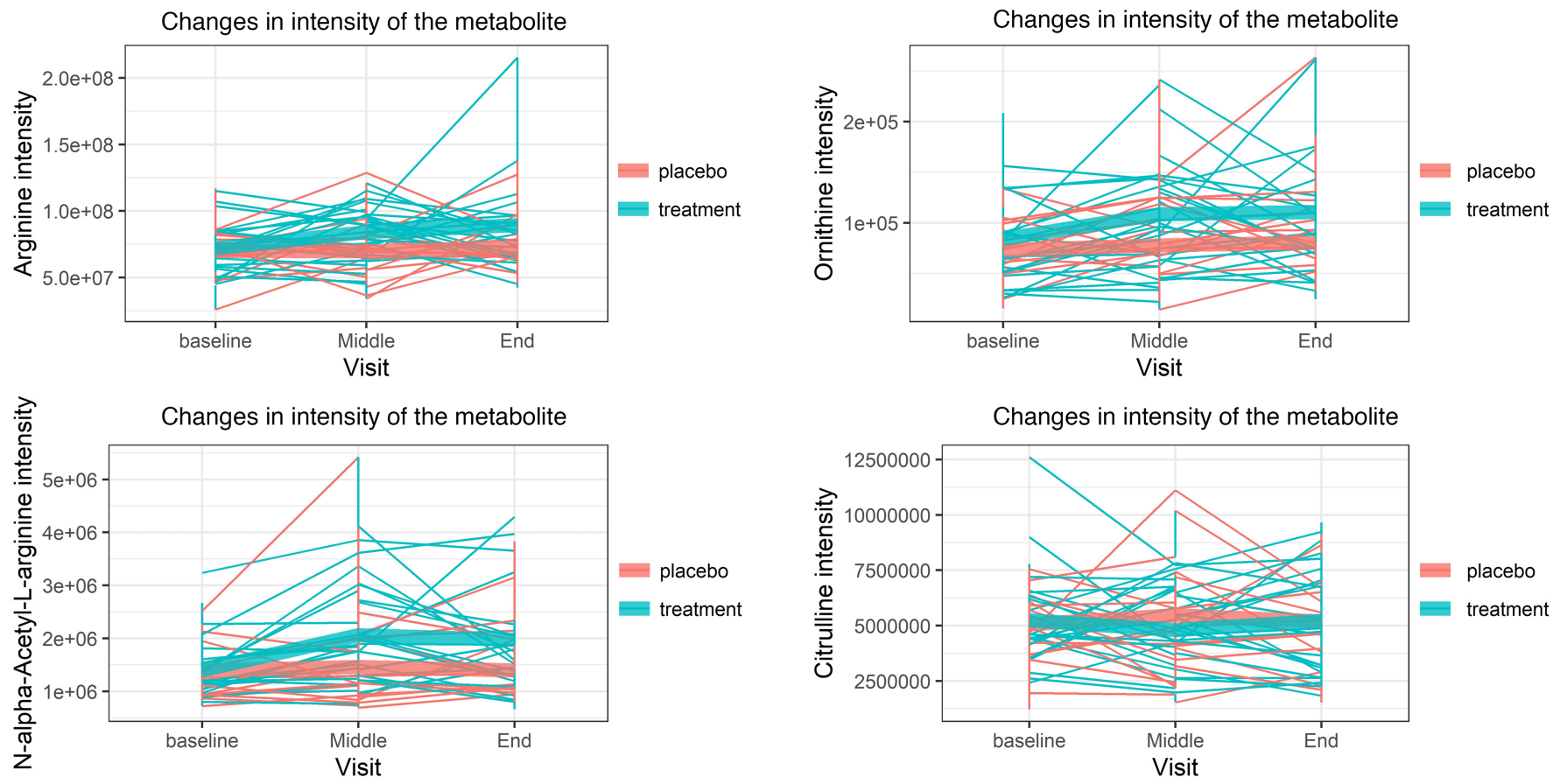

Figure 5. Plasma arginine pathway metabolites during treatment. Change in pattern of arginine-related pathway metabolites in the treatment versus placebo phase. $n=46$.

of DPP4 are increased in obese individuals $(31,32)$. CD26 inhibitors ameliorate airway inflammation in mouse models of asthma (33), perhaps through its roles in T cell function and glucose homeostasis. We found a change in the pattern of circulating dipeptides in plasma, which may reflect differential activity of DPP4 and other dipeptidases on inflammatory cytokines or regulators of glucose homeostasis such as GLP1. Further study of the effect of amino acid supplementation on these dipeptides and peptidase activity may clarify this pattern further.

One limitation of predictive metabolic biomarker profiling in this study is the sample size. Although an adequate number of participants was enrolled, based on our power calculation, the sample size for identifying the responders is relatively small, and we did not have a replication cohort to validate our identified predictors. Across all study visits, arginine-related metabolites showed no significant associations with ACT scores or lung function. However, higher citrulline levels were associated with higher FeNO measurements, suggesting a possible shift in L-arginine metabolism in severe asthma. These findings are supported by a previous study (7) finding higher expression of iNOS in those with a high-FeNO asthma phenotype, which produces NO and citrulline from arginine. L-Arginine is metabolized to NO via several NOS isoforms (neuronal NOS [nNOS], iNOS, and endothelial NOS [eNOS]), which can act in both beneficial and inflammatory capacities. Arginine can also be hydrolyzed through arginase, leading to the formation of urea, L-ornithine, and L-proline, which are precursors to polyamines and proline and may contribute to airway remodeling. In the lung, it has been proposed that increased mitochondrial arginine metabolism suppresses key signaling events that are significant in asthma pathogenesis.

Human airway epithelial cells respond adversely to ADMA via reduced NOx production, but this can be restored with L-citrulline treatment (34). The balance among arginine, ADMA, citrulline, and the regulation of NOS is unclear. It is difficult to find an L-arginine therapeutic range given the complexity of L-arginine metabolism. Oral supplementation with a combination of L-citrulline and L-arginine increased plasma L-arginine levels more effectively than treatment with L-arginine or L-citrulline alone (35). AAI [defined herein as arginine/(citrulline + ornithine)], which was identified in a post hoc analysis, may be a good measure of supplement effect. We found that an increased AAI was associated with lower FeNO and fewer exacerbation events, which may correlate with better asthma control. Currently available biomarkers for asthma severity include peripheral blood eosinophil counts, FeNO, and blood IgE levels. However, these biomarkers have limited use, as they are more suitable as biomarkers for type 2 asthma. 
Table 5. Top 10 metabolites with different temporal profiles in FeNO groups during treatment period

\begin{tabular}{cc}
\hline Metabolites & ${\text { Hotelling's } \mathbf{T}^{2}}^{2}$ \\
Taurine & 16.27 \\
Gly-Val & 15.67 \\
Creatine & 12.82 \\
Hypoxanthine & 12.39 \\
Maltotriose & 12.38 \\
Xanthine & 12.32 \\
Lyxose & 12.27 \\
Ribose A & 12.19 \\
Xylose & 12.01 \\
\hline Orotic acid & 11.85
\end{tabular}

Our study population was a mixture of high-FeNO (characteristic of a type 2 asthma phenotype) and low-FeNO (characteristic of a non-type 2 asthma phenotype), and our AAI correlated with both asthma exacerbation events and FeNO from both groups in the study population. This finding implied that L-arginine supplementation may be beneficial in a subset of the patients regardless of their baseline FeNO status. Another implication of our findings regarding AAI levels is that future studies may focus on how to increase this index with supplementation of L-arginine, L-citrulline, or a combination of L-arginine and L-citrulline for improving asthma control.

Our study also provides a snapshot of the different metabolic profiles in high- and low-FeNO groups. The top metabolite, cystine, has been found to interact with NO. For example, NO can increase cystine uptake and elevate the intracellular glutathione level (36). Cysteine (cystine is its oxidized dimer form) was found to cooperate with $\mathrm{NO}$ and mediates the activation of soluble guanylate cyclase, which has relevance to the NO/cGMP signaling pathway (37). In future studies, metabolites in addition to those found to differentiate between FeNO and treatment status in the present study may provide clues allowing generation of new hypotheses regarding the relationship among NO, arginine, and metabolites.

Previous studies have included metabolite measurements at single time points, which may be misleading, as metabolite levels are very dynamic and affected largely by diet and environmental conditions. Our study provided a more consistent longitudinal approach compared with previous studies, so we were able to outline a longitudinal relationship between groups of metabolites and asthma-related clinical outcomes. The limitation of the longitudinal metabolic analysis is that the findings of the metabolomics results are semiquantitative, instead of quantitative concentrations. While the association between the metabolites and outcomes is not affected by this approach, we could not establish exact metabolite concentrations that may affect clinical outcomes. This will need to be addressed with future studies.

Table 6. Top 10 metabolites with different temporal profiles in response versus nonresponse during treatment period

\begin{tabular}{cc} 
Metabolite & Hotelling's $\boldsymbol{T}^{\mathbf{2}}$ \\
\hline Vanillin & 18.31 \\
\hline 4-Guanidinobutyric.acid & 15.25 \\
Benzoic acid & 14.03 \\
\hline Cyclohexanamine & 13.48 \\
\hline Bis(2,2,6,6-tetramethyl-4-piperidyl) sebacate & 13.43 \\
\hline 4-Nitrophenol & 12.30 \\
\hline Benzoic acid, 4-hydroxy- & 11.81 \\
\hline 3-Aminosalicylic acid & 11.46 \\
Methyltestosterone & 11.03 \\
\hline
\end{tabular}


Additional limitations deserve mention. For one, the failure to demonstrate a difference in the primary end point may simply be due to the small sample size in this single-center, crossover study. Another possible explanation may lie in the fact that we do not have a reliable biomarker to predict which patient cohorts will respond to L-arginine therapy. In our study, we used an untargeted metabolomic approach for analyzing plasma metabolites sampled at each visit. We identified 2 metabolites, $\mathrm{PGH}_{2}$ and $N_{\alpha}$-acetyl-L-arginine, that can be used as biomarkers to predict L-arginine treatment response in future studies. However, further validation of $\mathrm{PGH}_{2}$ using more specific methodology for prostaglandin analysis, and an authentic $\mathrm{PGH}_{2}$ standard, is needed. Last, our study design was complex. The assignment of severe asthma patients to a high- or low-FeNO group at baseline before initiation of a crossover design study required that we use a rigorous statistical approach that included the use of interaction terms. We recognize that this adds a layer of complexity to the data.

Although there was no overall significant clinical benefit of L-arginine supplementation in our severe asthma cohort, we did identify a subgroup of subjects that may benefit from the intervention. Future clinical trial design should incorporate subjects' baseline metabolomics and L-arginine levels during enrollment. Our study is a first step in clarifying both the right study population and metabolic biomarkers needed to identify true responders. This study also provides an overview of arginine-related metabolism and how it is related to clinical outcomes using a longitudinal approach. Further work will clarify the relationship between L-arginine and L-citrulline in vivo with exact concentration measurements. This will aid in the clarification of the biomarker profiles and metabolic fingerprints most likely to respond to L-arginine supplementation.

\section{Methods}

Study design and participants. We designed a randomized, double-blind, placebo-controlled, crossover trial to evaluate the efficacy of oral L-arginine as a supplementary treatment for adults with severe asthma (ClinicalTrials.gov NCT01841281). The design was a 2-group study stratifying participants by FeNO levels with the low-FeNO (or normal-FeNO) group defined as a concentration $<20 \mathrm{ppb}$, and the high-FeNO group defined as having FeNO >25 ppb. If a subject had an initial FeNO level that fell between these levels, they could be rescreened 4 weeks later and reclassified. The patients were recruited primarily from severe asthma referral clinics within the University of California-Davis Asthma Network (UCAN) clinic.

Adult patients (18 years or older) with severe asthma who met prior American Thoracic Society (ATS) criteria for the definition of severe asthma (38) were included in the study. The detailed inclusion and exclusion criteria are provided in Supplemental Methods.

Procedures and randomization. After informed consent and a screening visit to determine eligibility and baseline FeNO, subjects were randomized into the 30-week crossover study trial: 12 weeks to treatment A (L-arginine or placebo), followed by a 6-week washout period, and 12 weeks of treatment B. We treated individuals with severe asthma with L-arginine at a dose of $0.05 \mathrm{~g} / \mathrm{kg}$ twice a day $(6-10 \mathrm{~g} / \mathrm{d})$ based on ideal body weight, or a matching placebo. The dose was determined based on our pilot study (39). Drug and placebo were provided by Jarrow Pharmaceuticals, and an Investigational New Drug (IND) application for the formulation was approved by the FDA (L-arginine in severe asthma 14420). The study visits were performed at the Clinical Research Center, which is part of the UC Davis Clinical and Translational Science Center. Participants were asked to record daily morning and evening peak flow rates, albuterol use, and steroid use in a written log. These data were reviewed at each study visit. All patients were on standard controller therapy, including appropriate doses of inhaled corticosteroids and long-acting bronchodilators. The randomization process and disbursement of the L-arginine and matching placebo was managed by the UC Davis IDS.

Outcomes. The primary end point for the study was a composite end point for a total number of "moderate" asthma exacerbations during the 12-week period of L-arginine intervention. We used the ATS/European Respiratory Society (ERS) statement for asthma exacerbations that was current in 2009 when this study was conceived (40). These events included the following singular end points: (a) a drop in morning peak expiratory flow $(\mathrm{PEF})>30 \%$ from baseline on 2 consecutive days; (b) need for initiation of oral steroids or increased dose of inhaled corticoid steroids in the morning on any 2 consecutive days; and (c) doubling of short-acting $\beta$-agonist use (e.g., number of puffs of albuterol/day for 2 consecutive days) (41-43). The secondary clinical end points were recorded at each of the 6 study visits. These included: asthma control test score, lung function, and weight. Phenotyping was by FeNO rather than sputum analysis for eosinophil and neutrophil numbers (44).

Plasma untargeted metabolomics analysis. Plasma $(20 \mu \mathrm{L})$ was extracted for HILIC-QEHFMS (hydrophilic interaction chromatography via Q Exactive HF Hybrid Quadrupole-Orbitrap Mass Spectrometer; Thermo Fisher Scientific) analysis, and $30 \mu \mathrm{L}$ plasma was extracted and analyzed using gas 
chromatography/time-of-flight mass spectrometry (GC-TOFMS) analysis as previously described (45). Identification and confidence scoring of all annotations were performed as reported in Supplemental Methods. With the range of annotation confidence scores obtained from untargeted profiling, it is important to perform additional validation studies. Therefore, we openly report our results and note confidence levels when appropriate. Full methods are available in Supplemental Methods.

Statistics. For a sample size of 50 subjects, we would have $95 \%$ power to detect a 0.6 standard deviation change in the lung functions including $\mathrm{FEV}_{1}$ and FVC at $\alpha=0.025$ accounting for a $10 \%$ attrition rate. Details about the power calculation are provided in Supplemental Methods. Data were analyzed on an intentionto-treat basis, and the significance level was defined by a $P$ value less than 0.05 . SAS 9.4 (SAS Institute) was used for studying effects of L-arginine supplementation on clinical end points. A generalized linear mixed model was used for analysis, accounting for correlated repeated measurements, which enabled us to account for expected missing data points (46). The distribution of exacerbation events was assumed to be a Poisson distribution. For the primary hypothesis, we included an indicator in the model that allowed for the subjects to be on active treatment. We first examined the carryover and period effects, then tested for the treatment effect and examined the interaction term between treatment and FeNO. For secondary clinical outcomes, we performed the same analysis, except we used a linear mixed-effects model for the continuous end points. The next step was to identify baseline metabolites as predictors for treatment response. We defined treatment response as a reduction in exacerbation events by $33 \%$ during L-arginine treatment. The individual event reduction was calculated as follows: (events during treatment period - events during the placebo period)/(events during the placebo period). Subjects with 0 events in the placebo period or those who did not complete both treatment and placebo phases were excluded. The baseline metabolomic profiling (first visit) was used for the downstream analysis. PLS-DA and hierarchical cluster analysis (using Euclidean as similarity measure and Ward's linkage as clustering algorithm) were performed using MetaboAnalyst 4.0 (47) to identify the predictive metabolites. A linear mixed-effects model was used to test the association between longitudinal metabolite levels and clinical outcomes, including FeNO at each visit. Metabolite pattern changes between 2 biological conditions were tested using the method of MEBA time-series analysis (24) built in MetaboAnalyst 4.0 and included (a) treatment versus placebo; (b) high- versus low-FeNO groups during the treatment period; (c) and treatment response versus nonresponse groups during the treatment period. Hotelling's $T^{2}$ statistics were used to rank the metabolites with different temporal profiles between each of the 2 biological conditions under study.

Study approval. Written informed consent was received from participants before inclusion in the study. The study was approved by the IRB committee at UCD. The Data and Safety Monitoring Board approved by the NIH reviewed study progress and participant safety, and ensured appropriate data management and analysis.

\section{Author contributions}

SYL, ALL, MR Showalter, AAZ, and NJK wrote the manuscript. SYL and LQ developed the analysis plan, and LQ supervised SYL, YL, and MR Showalter with the data analysis. ALL, LQ, and NJK designed the study and wrote the grant. ALL, MR Sa, MR Showalter, and ZAK performed sample handling and metabolomic profiling. ALL, LF, and CK collected clinical data and specimens. NJK, OF, AAZ, and LQ supervised the research.

\section{Acknowledgments}

This study was supported by NIH grants R01HL105573, DK097154, UL1TR001860, and K08HL114882; and a grant from TRDRP. The investigators thank the members of the Data Safety and Monitoring Board for their oversight and commitment to patient safety.

Address correspondence to: Nicholas Kenyon, Division of Pulmonary and Critical Care Medicine, $4150 \mathrm{~V}$ Street, Suite 3400, Sacramento, California 95817, USA. Phone: 916.734.3564; Email: njkenyon@ucdavis.edu.

SYL's present address is: Department of Medicine, University of Colorado Anschutz Medical Campus, Aurora, Colorado, USA.

MR Sa's present address is: California Northstate University College of Medicine, Elk Grove, California, USA. 
1. Schneider A, et al. Diagnosing asthma in general practice with portable exhaled nitric oxide measurement — results of a prospective diagnostic study. Respir Res. 2009;10:15.

2. Dweik RA, et al. An official ATS clinical practice guideline: interpretation of exhaled nitric oxide levels (FENO) for clinical applications. Am J Respir Crit Care Med. 2011;184(5):602-615.

3. Wang Z, et al. The Clinical Utility of Fractional Exhaled Nitric Oxide (FeNO) in Asthma Management. Rockville, Marlyand, USA Agency for Healthcare Research and Quality; 2017. AHRQ publication 17(18)-EHC030-EF. https://doi.org/10.23970/ AHRQEPCCER197. December 2017. Accessed June 17, 2020.

4. Kenyon NJ, Bratt JM, Linderholm AL, Last MS, Last JA. Arginases I and II in lungs of ovalbumin-sensitized mice exposed to ovalbumin: sources and consequences. Toxicol Appl Pharmacol. 2008;230(3):269-275.

5. Bratt JM, Franzi LM, Linderholm AL, Last MS, Kenyon NJ, Last JA. Arginase enzymes in isolated airways from normal and nitric oxide synthase 2-knockout mice exposed to ovalbumin. Toxicol Appl Pharmacol. 2009;234(3):273-280.

6. Bratt JM, Franzi LM, Linderholm AL, O'Roark EM, Kenyon NJ, Last JA. Arginase inhibition in airways from normal and nitric oxide synthase 2-knockout mice exposed to ovalbumin. Toxicol Appl Pharmacol. 2010;242(1):1-8.

7. Xu W, et al. Arginine metabolic endotypes related to asthma severity. PLoS One. 2017;12(8):e0183066.

8. Xu W, et al. Increased mitochondrial arginine metabolism supports bioenergetics in asthma. J Clin Invest. 2016;126(7):2465-2481

9. Wu G, et al. Arginine metabolism and nutrition in growth, health and disease. Amino Acids. 2009;37(1):153-168.

10. Benson RC, Hardy KA, Morris CR. Arginase and arginine dysregulation in asthma. J Allergy (Cairo). 2011;2011:736319.

11. Bahadoran Z, Mirmiran P, Tahmasebinejad Z, Azizi F. Dietary L-arginine intake and the incidence of coronary heart disease: Tehran lipid and glucose study. Nutr Metab (Lond). 2016;13:23.

12. Pahlavani N, et al. L-arginine supplementation and risk factors of cardiovascular diseases in healthy men: a double-blind randomized clinical trial. F1000Res. 2014;3:306.

13. Morris CR, et al. A randomized, placebo-controlled trial of arginine therapy for the treatment of children with sickle cell disease hospitalized with vaso-occlusive pain episodes. Haematologica. 2013;98(9):1375-1382.

14. Bratt JM, Zeki AA, Last JA, Kenyon NJ. Competitive metabolism of L-arginine: arginase as a therapeutic target in asthma. J Biomed Res. 2011;25(5):299-308.

15. Linderholm AL, Bratt JM, Schuster GU, Zeki AA, Kenyon NJ. Novel therapeutic strategies for adult obese asthmatics. Immunol Allergy Clin North Am. 2014;34(4):809-823.

16. Sibal L, Agarwal SC, Home PD, Boger RH. The role of asymmetric dimethylarginine (ADMA) in endothelial dysfunction and cardiovascular disease. Curr Cardiol Rev. 2010;6(2):82-90.

17. Holguin F, et al. L-Citrulline increases nitric oxide and improves control in obese asthmatics. JCI Insight. 2019;4(24):e131733.

18. Mabalirajan U, et al. Beneficial effects of high dose of L-arginine on airway hyperresponsiveness and airway inflammation in a murine model of asthma. J Allergy Clin Immunol. 2010;125(3):626-635.

19. Yang Y, et al. Safety of long-term dietary supplementation with L-arginine in rats. Amino Acids. 2015;47(9):1909-1920.

20. Zhang R, et al. 1-Arginine administration attenuates airway inflammation by altering 1-arginine metabolism in an NC/Nga mouse model of asthma. J Clin Biochem Nutr. 2015;56(3):201-207.

21. Oliw EH, Stark K, Bylund J. Oxidation of prostaglandin $\mathrm{H}(2)$ and prostaglandin $\mathrm{H}(2)$ analogues by human cytochromes $\mathrm{P} 450$ : analysis of omega-side chain hydroxy metabolites and four steroisomers of 5-hydroxyprostaglandin I(1) by mass spectrometry. Biochem Pharmacol. 2001;62(4):407-415.

22. Meyerholz DK, Lambertz AM, McCray PB. Dipeptidyl peptidase 4 distribution in the human respiratory tract: implications for the Middle East Respiratory syndrome. Am J Pathol. 2016;186(1):78-86.

23. Nieto-Fontarigo JJ, González-Barcala FJ, San José E, Arias P, Nogueira M, Salgado FJ. CD26 and asthma: a comprehensive review. Clin Rev Allergy Immunol. 2019;56(2):139-160.

24. Tai YC, Speed TP. On gene ranking using replicated microarray time course data. Biometrics. 2009;65(1):40-51.

25. Scott JA, North ML, Rafii M, Huang H, Pencharz P, Grasemann H. Plasma arginine metabolites reflect airway dysfunction in a murine model of allergic airway inflammation. J Appl Physiol. 2015;118(10):1229-1233.

26. Kharitonov SA, Sapienza MA, Barnes PJ, Chung KF. Prostaglandins E2 and F2alpha reduce exhaled nitric oxide in normal and asthmatic subjects irrespective of airway caliber changes. Am J Respir Crit Care Med. 1998;158(5 pt 1):1374-8.

27. Asosingh K, et al. Arginine metabolic control of airway inflammation. JCI Insight. 2020;5(2):e127801.

28. Liang D, et al. Perturbations of the arginine metabolome following exposures to traffic-related air pollution in a panel of commuters with and without asthma. Environ Int. 2019;127:503-513.

29. Xu J, et al. Dipeptidyl peptidase IV (DPP-4) inhibition alleviates pulmonary arterial remodeling in experimental pulmonary hypertension. Lab Invest. 2018;98(10):1333-1346.

30. Zou H, Zhu N, Li S. The emerging role of dipeptidyl-peptidase-4 as a therapeutic target in lung disease. Expert Opin Ther Targets. 2020;24(2):147-153

31. Nistala R, Savin V. Diabetes, hypertension, and chronic kidney disease progression: role of DPP4. Am J Physiol Renal Physiol. 2017;312(4):F661-F670

32. Lamers D, et al. Dipeptidyl peptidase 4 is a novel adipokine potentially linking obesity to the metabolic syndrome. Diabetes. 2011;60(7):1917-1925.

33. Stephan M, et al. Effects of dipeptidyl peptidase-4 inhibition in an animal model of experimental asthma: a matter of dose, route, and time. Physiol Rep. 2013;1(5):e00095.

34. Winnica D, Que LG, Baffi C, Grasemann H, Fiedler K, Yang Z, et al. L-Citrulline prevents asymmetric dimethylarginine-mediated reductions in nitric oxide and nitrosative stress in primary human airway epithelial cells. Clin Exp Allergy. 2017;47(2):190-9.

35. Suzuki T, Morita M, Hayashi T, Kamimura A. The effects on plasma L-arginine levels of combined oral L-citrulline and L-arginine supplementation in healthy males. Biosci Biotechnol Biochem. 2017;81(2):372-375.

36. Li H, Marshall ZM, Whorton AR. Stimulation of cystine uptake by nitric oxide: regulation of endothelial cell glutathione levels. Am J Physiol. 1999;276(4):C803-C811.

37. Fernhoff NB, Derbyshire ER, Marletta MA. A nitric oxide/cysteine interaction mediates the activation of soluble guanylate cyclase. Proc Natl Acad Sci U S A. 2009;106(51):21602-21607. 
38. [No authors listed]. Proceedings of the ATS workshop on refractory asthma: current understanding, recommendations, and unanswered questions. American Thoracic Society. Am J Respir Crit Care Med. 2000;162(6):2341-2351.

39. Kenyon NJ, Last M, Bratt JM, Kwan VW, O'Roark E, Linderholm A. L-Arginine supplementation and metabolism in asthma. Pharmaceuticals (Basel). 2011;4(1):187-201.

40. Reddel HK, et al. An official American Thoracic Society/European Respiratory Society statement: asthma control and exacerbations: standardizing endpoints for clinical asthma trials and clinical practice. Am J Respir Crit Care Med. 2009;180(1):59-99.

41. Green $\mathrm{RH}$, et al. Asthma exacerbations and sputum eosinophil counts: a randomised controlled trial. Lancet. 2002;360(9347):1715-1721.

42. Green RH, Brightling CE, Woltmann G, Parker D, Wardlaw AJ, Pavord ID. Analysis of induced sputum in adults with asthma: identification of subgroup with isolated sputum neutrophilia and poor response to inhaled corticosteroids. Thorax. 2002;57(10):875-879.

43. Pauwels RA, et al. Effect of inhaled formoterol and budesonide on exacerbations of asthma. Formoterol and Corticosteroids Establishing Therapy (FACET) International Study Group. N Engl J Med. 1997;337(20):1405-1411.

44. Carr TF, Zeki AA, Kraft M. Eosinophilic and noneosinophilic asthma. Am J Respir Crit Care Med. 2018;197(1):22-37.

45. Barupal DK, et al. A Comprehensive plasma metabolomics dataset for a cohort of mouse knockouts within the International Mouse Phenotyping Consortium. Metabolites. 2019;9(5):E101.

46. Zeger SL, Liang KY. Longitudinal data analysis for discrete and continuous outcomes. Biometrics. 1986;42(1):121-130

47. Chong J, et al. MetaboAnalyst 4.0: towards more transparent and integrative metabolomics analysis. Nucleic Acids Res. 2018;46(W1):W486-W494. 